\title{
Two-Sided Matching on Comprehensive Transportation Network Emergency Vehicles' Allocation
}

\author{
Kunwei Xie $\mathbb{D}^{1},{ }^{1}$ Heying Xu $\mathbb{D}^{1},{ }^{1}$ and Hongxia $\mathrm{Lv} \mathbb{D i D}^{1,2,3}$ \\ ${ }^{1}$ School of Transportation and Logistics, Southwest Jiaotong University, Chengdu 610031, China \\ ${ }^{2}$ National and Local Joint Engineering Laboratory of Comprehensive Intelligent Transportation, Southwest Jiaotong University, \\ Chengdu 610031, China \\ ${ }^{3}$ National Engineering Laboratory of Integrated Transportation Big Data Application Technology, Chengdu 610031, China \\ Correspondence should be addressed to Heying Xu; 380013157@qq.com
}

Received 5 April 2021; Revised 27 May 2021; Accepted 17 June 2021; Published 5 July 2021

Academic Editor: Chi-Hua Chen

Copyright (C) 2021 Kunwei Xie et al. This is an open access article distributed under the Creative Commons Attribution License, which permits unrestricted use, distribution, and reproduction in any medium, provided the original work is properly cited.

\begin{abstract}
In emergency rescue, the allocation of comprehensive transportation network emergency vehicles often affects the efficiency of the whole rescue process. In the context of disasters, this paper researches the one-to-many two-sided matching problem between the emergency vehicles and the materials to be transported. Firstly, based on the needs of both parties involved in the matching, the satisfaction evaluation systems are constructed; with the goal of maximizing the weighted satisfaction of the affected areas and vehicles, the optimization model of the materials and emergency vehicles matching is established; then, an improved National Intern Matching Program (NIMP) algorithm is designed to solve the model, which is based on the $k: 1$ experimental pairing and updating ideas, and can take into account the capacity and destination constraints of vehicles in the matching process. Finally, through the calculation of an example, the matching scheme can make the satisfaction of material transportation reach 0.7392 , and the simulation analysis proves that the scheme keeps certain stability in risky conditions.
\end{abstract}

\section{Introduction}

Emergency causes huge losses and social impact because of the suddenness, unpredictability, and diffusion. In the process of emergency response, transportation network often plays an important role in ensuring the transportation of materials and transfer of disaster victims. A scientific and reasonable allocation scheme of emergency vehicles can make limited transportation resources fit the transportation need between the supply point and the affected area to the maximum extent, which is of great significance for the improvement of emergency capacity of the whole society. However, disasters cause different degrees of damage to the road, resulting in one or more transportation networks getting paralyzed, which is not conducive to the emergency work.

With the rapid construction of transportation infrastructure and modern comprehensive transportation system, the division of labor among various modes of transportation becomes clearer and closer. Therefore, the formulation of emergency transportation schemes should be based on different needs of affected areas, different characteristics of demand materials, and different modes of transportation, to realize the reasonable and efficient utilization of the emergency vehicles. Comprehensive transportation network emergency vehicles refer to all kinds of comprehensive transportation mobile equipment that can be used in the territory and the whole rescue scope in the process of joint prevention and control, emergency rescue, disaster victims transfer, post disaster recovery, and other work in order to control the disaster and reduce losses under the constraints of certain fixed facilities and channel transport capacity.

Existing research on material transportation optimization mainly includes the emergency vehicle route planning, distribution, and scheduling.

On the emergency vehicle route planning, Zhang et al. [1] established the shortest path model considering the road 
conditions and the shortage of demand resources. Hu [2] used a linear programming model to plan the route. Özdamar et al. [3] built a combined transportation path model based on node clustering.

On the vehicle distribution, Rawls et al. [4] proposed a two-stage mixed integer programming location model. Bozorgi-Amiri et al. [5] established an emergency material distribution model considering the uncertainty of demand location.

On the vehicle scheduling, Afshar et al. [6], Sabouhi et al. [7], and Huang et al. [8] researched the emergency vehicle scheduling model from the perspective of logistics operation. Laporte et al. [9], Arda et al. [10], Duan, et al. [11], and Klibi et al. [12] proposed the multistage model to optimize vehicle scheduling.

The above papers mainly solve the problems of emergency vehicle distribution and scheduling, and the limitations are as follows. (1) Existing literature considers the same model of vehicles, and materials are homogeneous. (2) Existing literature usually ignores train, and flight operation is limited by timetable. Actually, in emergency transportation, different vehicles have different loading attributes and some of them may have fixed operating period. These constraints make existing research neither clarify the matching relationship between materials and vehicles nor put into use directly.

Considering that the two-sided matching theory can be used to solve the matching problem between two objects and the development of emergency management information platform makes it easier to bring the information of various rescue resources into the unified platform for centralized management, it is possible for us to research how to find a satisfactory matching between materials and vehicles with this theory.

Two-sided matching theory was first proposed by Gale and Shapley [13], and Roth [14, 15] clearly defined the concepts of "two-sided" and "two-sided matching." "Twosided" refers to the participants in the market who belong to two disjoint sets, and "matching" refers to the two-sided nature of market exchange. Since both parties have the preference to get stable matching, two-sided matching theory is used to study the matching process of disjoint parties with stable preference.

The process of emergency vehicles' allocation is participated by the vehicles and materials that need to be transported, and the core is to find an optimal resource allocation scheme in the context of emergency so that both sides can find the matching objects which meet their own needs. Therefore, combined with the characteristics of strong timeliness, high uncertainty, and weak economy of emergency rescue, this paper proposes an emergency vehicle allocation method based on the two-sided matching theory, which has three main contributions:

(1) The application of two-sided matching theory is extended

(2) A two-sided matching model considering materials' loading demands is established, which can guide the matching of materials with specific emergency vehicles

(3) An example validates the stability and operability of emergency vehicles' allocation scheme under certain risks

The remainder of the paper is organized as follows. Section 2 introduces the problem and presents an analysis on matching satisfaction. Section 3 formulates a two-side matching model, and an improved Nation Intern Matching Program (NIMP) algorithm is proposed in Section 4. Thereafter, a matching example is conducted in Section 5. Section 6 concludes the paper and provides potential future work.

\section{Matching Satisfaction Calculation}

2.1. Problem Presentation. Suppose that there are disaster affected areas and each area needs $M$ materials. We use $R$ to denote the set of materials to transport:

$$
\begin{aligned}
R & =\left\{R_{1}, R_{2}, \ldots, R_{i}, \ldots, R_{I}\right\}^{T}, \\
& =\left[\begin{array}{ccc}
r_{11} & \ldots & r_{1 M} \\
\vdots & \ddots & \vdots \\
r_{I 1} & \ldots & r_{I M}
\end{array}\right],
\end{aligned}
$$

where $R_{i}$ denotes the set of materials demand of area $i$, $R_{i}=\left\{r_{i 1}, \ldots, r_{i m}, \ldots, r_{i M}\right\}$, and $r_{i m}$ denotes the material $m$ required for area $i, i=1,2, \ldots, I$ and $m=1,2, \ldots M$.

Emergency vehicle set $V$ is composed of $J$ vehicles, $V=\left\{V_{1}, \ldots, V_{j}, \ldots, V_{J}\right\} . \quad V_{j}$ denotes vehicle $j$, $j=1,2, \ldots, J$.

Mapping $\mu R \cup S \longrightarrow R \cup S$ denotes the matching result between $r_{i m}$ and $V_{j}$. If the rim is transported by $V_{j}$, $r_{i m} \in \mu\left(V_{j}\right)$ and $V_{j} \in \mu\left(r_{i m}\right)$.

2.2. Model Assumptions. Before modeling, we make the following assumptions: (1) the materials needed for one disaster affected area can be transported by different vehicles; (2) each vehicle is only used to meet the transportation demand of one disaster area, but it can transport different materials needed in this area; (3) each material rim cannot be separated or transshipped during transportation; (4) if vehicle $V_{j}$ is also the required material for affected areas, we assume a virtual good $r_{i m}$ in set $R$, and this $r_{i m}$ has the following characteristics: $r_{i m}=V_{j}$; the weight of $r_{i m}$ is $0 ; r_{i m}$ can only be transported by $V_{j}$; (5) the number and capacity of available vehicles are limited.

Because decision makers can obtain the information of the affected areas' material demand and comprehensive transportation network emergency vehicles through the emergency management information platform, there is a two-sided matching market between the demand materials and emergency vehicles based on the information platform. According to the model assumptions, this is a one-to-many two-sided matching market. One affected area in this market can be served by multiple vehicles, which forms a one-to- 
many matching relationship between one area and vehicles; at the same time, one vehicle can transport a variety of materials, so that the matching market between vehicles and materials can be regarded as one-to-many matching relationships restricted by the materials' destination. The operating mechanism of this matching market is shown in Figure 1.

2.3. Matching Satisfaction Index Systems. The premise of establishing the demand materials and emergency transportation vehicles' matching optimization model is the construction of the evaluation index systems on both sides so that we can evaluate the satisfaction of different matching schemes. The parameters related to evaluation models are given in Table 1.

We use $U=\{u 1, u 2, \ldots, u 4\}$ to denote the satisfaction evaluation index set of demand materials and $S=\{s 1, s 2, \ldots, s 4\}$ to denote the satisfaction evaluation index set of comprehensive transportation network emergency vehicles. Combined with the research on the evaluation of emergency material allocation [16-20] and vehicle-material matching problem [21-25] and considering the potential applications of the proposed method in high-speed rail systems $[26,27]$, the evaluation index systems on both sides are established.

For each material to be transported, the optimal scheme is to make the transportation time as short as possible. And, the transportation route should be reliable, so as to avoid the routes damage making the material transportation interruption. Therefore, on the basis of the loading characteristics of materials, we establish the material satisfaction evaluation index system as follows.

(1) Arrival time index $u_{1}^{i m, j}$ : in emergency transportation, higher satisfaction of materials means earlier arrival time transport by vehicles:

$$
u_{1}^{i m, j}=\frac{T_{i m}^{\min }}{T_{i m}^{j}} .
$$

(2) Delay probability index $u_{2}^{i m, j}$ : the delay probability of $V_{j}$ reflects the reliability of vehicle route. Higher material satisfaction requires higher reliability and lower delay probability:

$$
u_{2}^{i m, j}=\frac{\xi_{j}^{\min }}{\xi_{j}} .
$$

(3) Loading condition index $u_{3}^{i m, j}$ : different vehicles have different loading conditions, such as thermal, container, and flat, and materials evaluate different vehicles according to their own requirements:

$$
u_{3}^{i m, j} \in[0,1] \text {. }
$$

(4) Cargo capacity index $u_{4}^{i m, j}$ : the weight of materials should not exceed the vehicles' weight limitations:

$$
u_{4}^{i m, j}= \begin{cases}0, & g_{i m}>c_{j}, \\ 1, & g_{i m} \leq c_{j} .\end{cases}
$$

The satisfaction value of the materials in relation to vehicles is calculated as follows. In equation (6), $\omega_{p}^{i m}$ denotes the weight of $u_{p}^{i m, j}, \sum_{p=1}^{4} \omega_{p}^{i m}=1$, and the satisfaction value matrix is $R_{V}, R_{V}=\left(\lambda_{j i m}\right)_{J \times I \times M}$ :

$$
\lambda_{j i m}=\prod_{p=1}^{4}\left(u_{p}^{i m, j}\right)^{\omega_{p}^{i m}}, \quad \forall j .
$$

The rescue mission of each vehicle is to deliver materials to the respective destinations reliably and effectively. In actual rescue, emergencies usually attack routes thus prolonging the transportation time. So, we introduce control coefficient $\kappa$ of arrival time, in response to the risk that materials cannot be delivered on schedule. Combined with vehicle loading operation, we establish the vehicle satisfaction evaluation index system as follows.

(1) Satisfaction time window index $s_{1}^{j, i m}$ : the arrival time of emergency transportation is usually uncertain. Therefore, we reserve satisfactory time window and keep a certain time margin to ensure the stability of the scheme:

$$
s_{1}^{j, i m}= \begin{cases}1, & T_{i m}^{j}+\kappa \cdot \widehat{T_{i m}^{j}} \leq T W_{i m}^{\min }, \\ 1-2\left(\frac{T_{i m}^{j}+\kappa \cdot \widehat{T_{i m}^{j}}-T W_{i m}^{\min }}{T W_{i m}^{\max }-T W_{i m}^{\min }}\right)^{2}, & T W_{i m}^{\min }<T_{i m}^{j}+\kappa \cdot \widehat{T_{i m}^{j}} \leq \frac{T W_{i m}^{\min }+T W_{i m}^{\max }}{2}, \\ 2\left(\frac{T_{i m}^{j}+\kappa \cdot \widehat{T_{i m}^{j}}-T W_{i m}^{\max }}{T W_{i m}^{\max }-T W_{i m}^{\min }}\right)^{2}, & \frac{T W_{i m}^{\min }+T W_{i m}^{\max }}{2}<T_{i m}^{j}+\kappa \cdot \widehat{T_{i m}^{j}} \leq T W_{i m}^{\max }, \\ 0, & T_{i m}^{j}+\kappa \cdot \widehat{T_{i m}^{j}}>T W_{i m}^{\max } .\end{cases}
$$




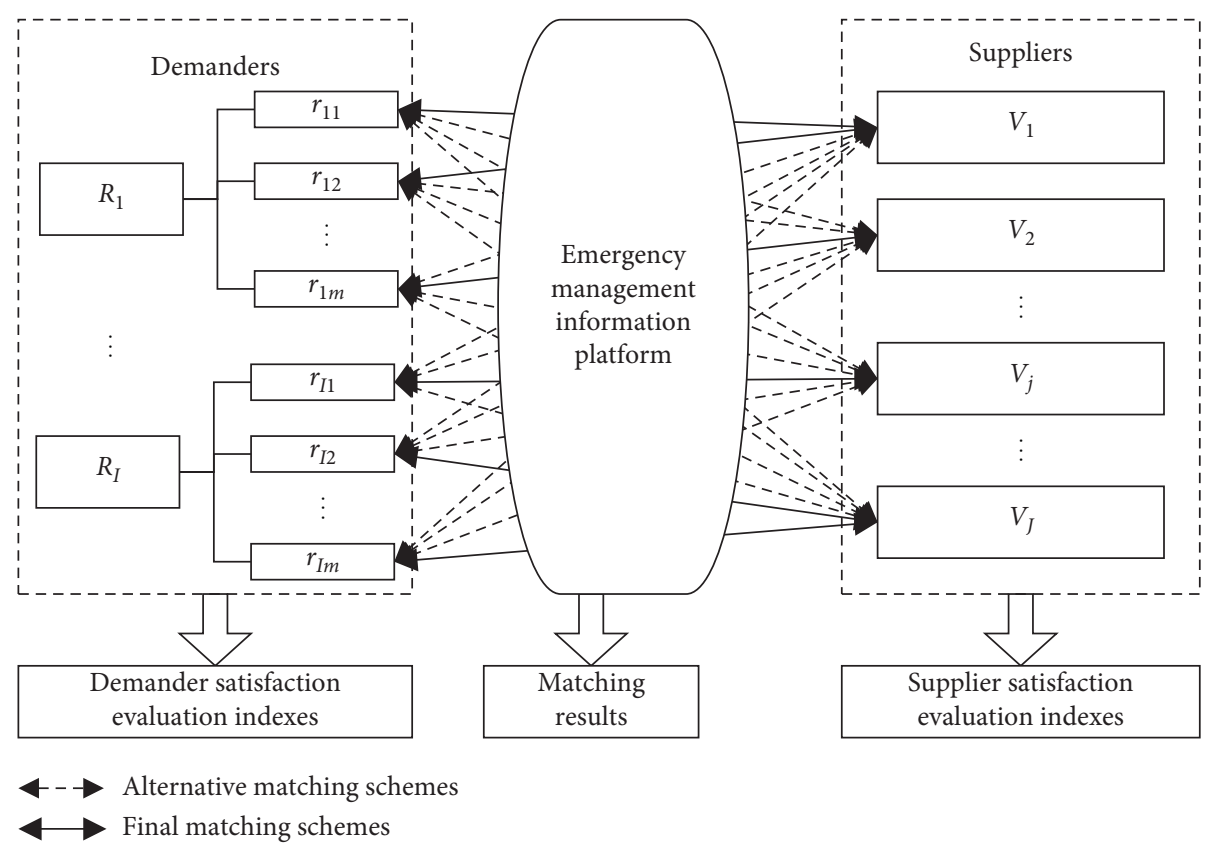

FIGURE 1: Two-sided matching mode of emergency platform.

TABle 1: Notation statement.

\begin{tabular}{|c|c|}
\hline Set & Statement \\
\hline $\begin{array}{l}U \\
S \\
l o_{j}\end{array}$ & $\begin{array}{l}\text { Set of demand materials satisfaction evaluation index } \\
\text { Set of emergency vehicles' satisfaction evaluation index } \\
\text { The loadable materials set of vehicle } V_{j}\end{array}$ \\
\hline $\begin{array}{l}\text { Parameter } \\
T_{i m}^{j} \\
T_{i m}^{\min } \\
T_{i m}^{j} \\
{\left[T W_{i m}^{\min }, T W_{i m}^{\max }\right]} \\
\xi_{j} \\
\xi_{j}^{\min } \\
d_{i m}^{j} \\
d_{j}^{\min }, d_{j}^{\max } \\
t_{i m}^{j} \\
t_{j}^{\min }, t_{j}^{\max } \\
g_{i m} \\
c_{j} \\
\kappa\end{array}$ & $\begin{array}{c}\text { Statement } \\
\text { Estimated arrival time of rim transported by } V_{j} \\
\text { Minimum value of } T_{i m}^{j} \text { for each } r_{i m} \\
\text { Maximum positive deviation of } T_{i m}^{j} \text { affected by emergency } \\
\text { Satisfaction arrival time window of } r_{i m} \\
\text { Delay probability of } V_{j} \\
\text { Minimum value of } \xi_{j} \\
\text { Deadhead kilometers of } r_{i m} \text { transported by } V_{j} \\
\text { Minimum and maximum value of } d_{i m}^{j} \text { for each } V_{j} \\
\text { Handling time of } r_{i m} \text { transported by } V_{j} \\
\text { Minimum and maximum value of } t_{i m}^{j} \text { for each } V_{j} \\
\text { Estimated weight of } r_{i m} \\
\text { Cargo capacity of } V_{j} \\
\text { Control coefficient of arrival time }\end{array}$ \\
\hline
\end{tabular}

(2) Empty driving index $s_{2}^{j, i m}$ : the origin of $V_{j}$ should be the same as the rim to avoid empty driving:

$$
s_{2}^{j, i m}=\frac{d_{j}^{\max }-d_{i m}^{j}}{d_{j}^{\max }-d_{j}^{\min }}+\varepsilon .
$$

In order to ensure materials are transported as many as possible, we use minimum $\varepsilon$ to make $s_{2}^{j, i m}>0$.

(3) Handling time index $s_{3}^{j, i m}$ : the handling time reflects the complexity of handling operations:

$$
s_{3}^{j, i m}= \begin{cases}0, & r_{i m} \notin l o_{j}, \\ \frac{t_{j}^{\max }-t_{i m}^{j}}{t_{j}^{\max }-t_{j}^{\min }+\varepsilon,} & r_{i m} \in l o_{j} .\end{cases}
$$

In addition, in order to ensure materials are transported as much as possible, we use minimum $\varepsilon$ to make $s_{2}^{j, i m}=0$ if and only if $r_{i m} \notin l o_{j}$. 
(4) Cargo capacity index $s_{4}^{j, i m}$ : the cargo capacity of vehicles should exceed the materials' weight:

$$
s_{4}^{j, i m}= \begin{cases}0, & g_{i m}>c_{j}, \\ 1, & g_{i m} \leq c_{j} .\end{cases}
$$

The satisfaction value of the vehicles in relation to materials is calculated as follows. In equation (11), $\omega_{q}^{j}$ denotes the weight of $s_{q}^{j, i m}, \sum_{q=1}^{4} \omega_{q}^{j}=1$, and the satisfaction value matrix is $V_{R}, V_{R}=\left(\theta_{j i m}\right)_{J \times I \times M}$ :

$$
\theta_{j i m}=\prod_{p=1}^{4}\left(s_{p}^{j, i m}\right)^{\omega_{q}^{j}}, \quad \forall i, m .
$$

\section{Two-Sided Matching Model}

Since different disaster affected areas have different urgency and materials play various roles in different areas, $\alpha_{i m}$ denoting demand urgency weight of rim is used to make the matching scheme satisfy the total material demand to the maximum extent. We aim at optimal satisfaction on both sides and establish a one-to-many two-sided matching model:

$$
\begin{aligned}
& \max F_{1}=\sum_{i=1}^{I} \sum_{m=1}^{M} \alpha_{i m} \cdot \sum_{j=1}^{J} \lambda_{j i m} \cdot x_{j i m} \\
& \max F_{2}=\sum_{i=1}^{I} \sum_{m=1}^{M} \sum_{j=1}^{J} \theta_{j i m} \cdot x_{j i m} \\
& \text { s.t. } \quad \sum_{j=1}^{J} x_{j i m} \leq 1, \quad(\forall i, m) \text {, } \\
& \left(\sum_{m=1}^{M} x_{j i m}\right) \cdot\left(\sum_{m=1}^{M} x_{j i, m}\right)=0, \quad\left(\forall j, i, i^{\prime} ; i \neq i^{\prime}\right) \\
& \sum_{i=1}^{I} \sum_{m=1}^{M} g_{i m} \cdot x_{j i m} \leq c_{j}, \quad(\forall j), \\
& \left(\lambda_{j i m}-\lambda^{\prime}\right) \cdot x_{j i m} \geq 0, \quad(\forall j, i, m), \\
& \left(\theta_{j i m}-\theta^{\prime}\right) \cdot x_{j i m} \geq 0, \quad(\forall j, i, m), \\
& x_{j i m}=1, \quad\left(\forall j, i ; r_{i m}=S_{j}\right), \\
& x_{j i m} \in\{0,1\}, \quad(\forall j, i, m) .
\end{aligned}
$$

Equations (12) and (13) are the object of the optimization model. Equation (12) means maximum satisfaction of affected areas. Equation (13) represents the optimal object of vehicles. Equation (14) limits one material matches at most one vehicle. Equation (15) indicates that one vehicle is only used to meet the transportation demand of one disaster area. Equation (16) gives the cargo capacity constraint, in which the cargo capacity of the vehicle should exceed the weight of matching materials. Equations (17) and (18) are the minimum matching satisfaction constraint: if and only if $r_{i m}$ and $\theta_{j i m}>\theta r$, matching between $r_{i m}$ and $V_{j}$ is possible. Equation (19) denotes if $r_{i m}=V_{j}, x_{j i m}=1$. Equation (20) denotes the range of decision variable $x_{j i m} . x_{j i m}=1$ denotes $r_{i m}$ matches $V_{j} \cdot x_{j i m}=0$ denotes $r_{i m}$ does not match $V_{j}$.

\section{Algorithm Design}

National Intern Matching Program (NIMP) algorithm is an efficient algorithm which has been applied in one-to-many matching problem and successfully served many markets. The NIMP is consisted of a matching phase and a tentative assignment and update phase. In the $k$ th step of the matching phase (which is called $k: 1$ step), the demandersupplier pairs such that the demander is top ranked on the supplier's ranking and the supplier is $k$ th ranked by the demander are sought to find (here we assume one supplier serves multiple demanders). If such matches are found, the algorithm proceeds to tentatively assign and update [28]. It is proved that NIMP algorithm can generate stable matching in given preference and obtain the optimal stable matching of one party under strict preference sequence.

Because there are two matching markets in the model: one vehicle matches multiple materials and one affected area matches multiple vehicles, and NIMP algorithm is used to solve only one matching market problem. This paper proposes an improved NIMP algorithm to solve the model. The definition of algorithm parameters is as follows.

$n$ denotes iteration variable; $R_{1}^{(n)}$ denotes unmatched set of materials in the nth iteration, $R_{2}^{(n)}$ denotes matched set of materials, $R_{1}^{(n)} \cap R_{2}^{(n)}=\varnothing, R_{1}^{(n)} \cup R_{2}^{(n)}=R ; P_{V j}^{(n)}$ is the set that $V_{j}$ is $n$th ranked by $r_{i m} \in P_{V_{j}}^{(n)}$, and $\lambda_{j i m}^{\prime}>\lambda^{\prime}, \theta_{j i m}>\theta^{\prime}$; $P_{V_{j}, i}^{(n)} \subseteq P_{V_{j}}^{(n)}$, each $r_{i m} \in P_{V_{j}, i}^{(n)}$ meets $\sum_{r_{i m} \in P_{V_{j}, i}^{(n)}} \lambda_{j i m}^{\prime}=\max$ $\sum_{r_{i m} \in\left(P_{V_{j}}^{(n)} \cap R_{i}\right)} \lambda_{j i m}^{\prime}, \sum_{r_{i m} \in P_{V_{j}, i}^{(n)}} g_{i m} \leq c_{j}$, and belongs to the same $R_{i} ; \mu^{(n)}$ denotes the matching scheme in the $n$th iteration; $c_{j}^{(n)}$ denotes the remaining cargo capacity of $V_{j}$ before nth iteration.

Step 1: calculate the satisfaction matrix $V_{R}=\left(\theta_{j i m}\right)_{J \times I \times M}$ and $R_{V}^{\prime}=\left(\lambda_{J}^{\prime} \times I \times M\right)=\left(\left(\lambda_{j i m}\right)_{J}\right.$ $\left.\cdot \alpha_{i m}\right) I \times M$, and obtain the preference order lists submitted by the demand materials and vehicles.

Step 2: initialize variables $n=1$. Determine whether $r_{i m}=V_{j}$ is present; if yes, $\mu^{(1)}\left(r_{i m}\right)=V_{j}$, $\mu^{(1)}\left(V_{j}\right)=r_{i m}, \quad R_{1}^{(1)}=R-\left\{r_{i m}\right\}, \quad$ and $R_{2}^{(1)}=R_{2}^{(1)}$ $\cup\left\{r_{\text {im }}\right\}$, and turn to step 3; else, $R_{1}^{(1)}=R, R_{2}^{(1)}=\varnothing$, $P_{V_{j}}^{(1)}=\varnothing, \mu^{(1)}\left(r_{i m}\right)=\varnothing, \mu^{(1)}\left(V_{j}\right)=\varnothing$, and turn to step 3.

Step 3: find the $n$th choice $V_{j}$ of each $r_{i m}$. Identify the set $P_{V_{j}}^{(n)}$ of $V_{j}, P_{V_{j}}^{(n)} \subseteq R_{1}^{(n)}$. If one material has the 
same satisfaction for different vehicles, take a random method to determine a virtual strict preference order. Match $V_{j}$ and $r_{i m}$ as the following steps.

(1) $j=1$.

(2) Determine whether $P_{V_{j}}^{(n)}=\varnothing$ is true; if yes, turn to step 3.8; else, turn to step (3).

(3) Determine whether $\mu^{(n)}\left(V_{j}\right)=\varnothing$ is true; if yes, turn to step (4); else, turn to step (3).

(a) Determine whether $\left|P_{V}^{(n)}\right|=1$ is true; if yes, let $\mu^{(n+1)}\left(r_{i m}\right)=V_{j}, \mu^{(n+1)}\left(V_{j}^{j}\right)=r_{i m}, R_{1}^{(n+1)}=R_{1}^{(n)}-$ $P_{V_{j}}^{(n)}, R_{2}^{(n+1)}=R_{2}^{(n)} \cup P_{V_{j}}^{(n)}$, and $c_{j}^{(n+1)}=c_{j}^{(n)}-g_{i m}$, and turn to step (8); else, turn to step (b).

(b) Determine whether there are different material need to be transported in the same direction in set $P_{V_{j}}^{(n)}$; if yes, turn to step (c); else, only if $\lambda_{j i m}^{\prime}=$ $\max \left\{\lambda_{\text {jim }}^{\prime}\right\}$, let $\mu^{(n+1)}\left(r_{\text {im }}\right)=V_{j}, \mu^{(n+1)}\left(V_{j}\right)=r_{i m}$, $R_{1}^{(n+1)}=R_{1}^{(n)}-P_{V_{j}}^{(n)}, \quad R_{2}^{(n+1)}=R_{2}^{(n)} \cup P_{V_{j}}^{(n)}, \quad$ and $c_{j}^{(n+1)}=c_{j}^{(n)}-g_{i m}$, and turn to step (8).

(d) Let $\mu^{(n+1)}\left(V_{j}\right)=P_{V_{j}, i}^{(n)}, \quad \mu^{(n+1)}\left(r_{i m}\right)=V_{j}$ $\left(r_{i m} \in P_{V_{j}, i}^{(n)}\right), R_{1}^{(n+1)}=R_{1}^{(n)}-P_{V_{j, i}}^{(n)}, R_{2}^{(n+1)}=R_{2}^{(n)} \cup$ $P_{V j, i}^{(n)} c_{j}^{(n+1)}=c_{j}^{(n)}-\sum_{m} g_{i m}$, and turn to step (8).

(4) Find the set $P_{V_{j}, i}^{(n)}$; determine whether $P_{V_{j}, i}^{(n)}$ is empty; if yes, turn to step (8); else turn to step (5). (5) Determine whether $P_{V_{j}, i}^{(n)}$ and $\mu^{(n)}\left(V_{j}\right)$ belong to the same $R_{i}$, if not, turn to step (6); if yes, let $\mu^{(n+1)}\left(V_{j}\right)=\mu^{(n)}\left(V_{j}\right) \cup P_{V_{j}, i}^{(n)}, \quad \mu^{(n+1)}\left(r_{i m}\right)=V_{j}$ $\left(r_{i m} \in P_{V_{j}, i}^{(n)}\right), \quad R_{1}^{(n+1)}=R_{1}^{(n)}-P_{V_{j, i}}^{(n)}, \quad R_{2}^{(n+1)}=R_{2}^{(n)}$ $\cup P_{V_{j, i}}^{(n)}, c_{j}^{(n+1)}=c_{j}^{(n)}-\sum_{m} g_{i m}$, and turn to step (8). (6) Determine whether $\quad \sum_{r_{i m} \in P_{V_{i}, i}^{(n)}} \lambda_{j i m}^{\prime}>$ $\sum_{r_{i m} \in \mu^{(n)}\left(V_{j}\right)} \lambda_{j i m}{ }^{\prime}$ is true; if yes, turn to step (7); if not, determine whether there is $r_{i m} \in P_{V_{j}}^{(n)}$ with the same destination as $r_{i m} \in \mu^{(n)}\left(V_{j}\right)$; if yes, let $\mu^{(n+1)}\left(V_{j}\right)=\mu^{(n)}\left(V_{j}\right) \cup P_{V_{j}, \mu^{(n)}\left(V_{j}\right)}^{(n)}, \quad \mu^{(n+1)}\left(r_{i m}\right)=$ $V_{j}\left(r_{i m} \in P_{V_{j}, i}^{(n)}\right), R_{1}^{(n+1)}=R_{1}^{(n)}-P_{V_{j,} \mu^{(n)}\left(V_{j}\right)}^{(n)}, R_{2}^{(n+1)}=$
$R_{2}^{(n)} \cup P_{V_{j} \mu^{(n)}\left(V_{j}\right)}^{(n)} c_{j}^{(n+1)}=c_{j}^{(n)}-\sum_{m} g_{i m}$, turn to step (8); else, turn to step (8).

(7) Let $R_{1}^{(n+1)}=R_{1}^{(n)} \cup \mu^{(n)}\left(V_{j}\right), \quad R_{2}^{(n+1)}=R_{2}^{(n)}-$ $\mu^{(n)}\left(V_{j}\right), P_{V_{j^{\prime}}}^{(n)}=P_{V_{j^{\prime}}}^{(n)} \cup \mu^{(n)}\left(V_{j}\right) \quad\left(V_{j}\right.$ is the $n$th choice of $\left.r_{i m}\right)$, recalculate $\mu^{(n)}\left(V_{j}\right)$; let $\mu^{(n+1)}\left(r_{i m}\right)=V_{j}\left(r_{i m} \in P_{V_{j}, i}^{(n)}\right), R_{1}^{(n+1)}=R_{1}^{(n+1)}-$ $P_{V_{j, i}}^{(n)}, \quad R_{2}^{(n+1)}=R_{2}^{(n+1)} \cup P_{V_{j, i}}^{(n)}, \quad$ and $\quad c_{j}^{(n+1)}=c_{j}^{(1)}$ $-\sum_{m} g_{i m}$, and turn to step (8).

(8) Determine whether $j \geq J$ is true; if yes, turn to step 4; else, $j=j+1$ and turn to step (2).

Step 4: determine whether $R_{1}^{(n)} \neq \varnothing$ is true; if yes, end the loop and turn to step 5; if not, determine whether $n \geq J$ is true; if not, $n=n+1$ and repeat steps 3-4; else, end the loop and turn to step 5.

Step 5: determine whether there are $\mu^{(n+1)}\left(V_{j 1}\right)$, $\mu^{(n+1)}\left(V_{j 2}\right)$, and $\mu^{(n+1)}\left(V_{j 3}\right)$ which satisfy that

(1) $\mu^{(n+1)}\left(V_{j 1}\right)$ and $\mu^{(n+1)}\left(V_{j 2}\right)$ belong to the same $R_{i}$ different from $\mu^{(n+1)}\left(V_{j 3}\right)$.

(2) $c_{j 1}^{(n+1)} \geq \sum_{r_{i m} \in \mu^{(n+1)}\left(V_{j 2}\right)} g_{i m}$.

(3) $\begin{array}{ll}\sum_{r_{i m} \in \mu^{(n+1)}\left(V_{j 3}\right)}, \lambda_{j 2 i m}^{\prime}-\sum_{r_{i m} \in \mu^{(n+1)}\left(V_{j, 3}\right)} & \lambda_{j 3 i m}^{\prime}> \\ & \sum_{r_{i m} \in \mu^{(n+1)}\left(V_{j 2}\right)} \lambda_{j 2 i m}-\sum_{r_{i m} \in \mu^{(n+1)}\left(V_{j 2}\right)} \lambda_{j 1 i m} .\end{array}$ $\mu^{(n+2)}\left(r_{i m}\right)=V_{j 1}\left(r_{i m} \in \mu^{(n+1)}\left(V_{j 2}\right)\right), \mu^{(n+2)}\left(V_{j 1}\right)=$ $\mu^{(n+1)}\left(V_{j 1}\right) \bigcup \mu^{(n+1)}\left(V_{j 2}\right), \quad \mu^{(n+2)}\left(V_{j 2}\right)=\mu^{(n+1)}$ $\left(V_{j 3}\right), \quad \mu^{(n+2)}\left(r_{i m}\right)=V_{j 2}\left(r_{i m} \in \mu^{(n+1)}\left(V_{j 3}\right)\right)$, $\mu^{(n+2)}\left(V_{j 3}\right)=\varnothing$, and output, else, output.

\section{Simulation Results and Analysis}

To verify the validity of the two-sided matching model, we use the data of 4 affected locations $\left(R_{1}, R_{2}, R_{3}, R_{4}\right)$, and each location needs 4 materials $\left(r_{11}, r_{12}, \ldots, r_{44}\right)$. At the same time, we collect information about 12 vehicles $\left(V_{1}, V_{2}, \ldots, V_{12}\right)$ used for emergency transportation. Information on materials and vehicles is shown in Tables 2 and 3 .

In addition, we calculate the estimated arrival time as follows:

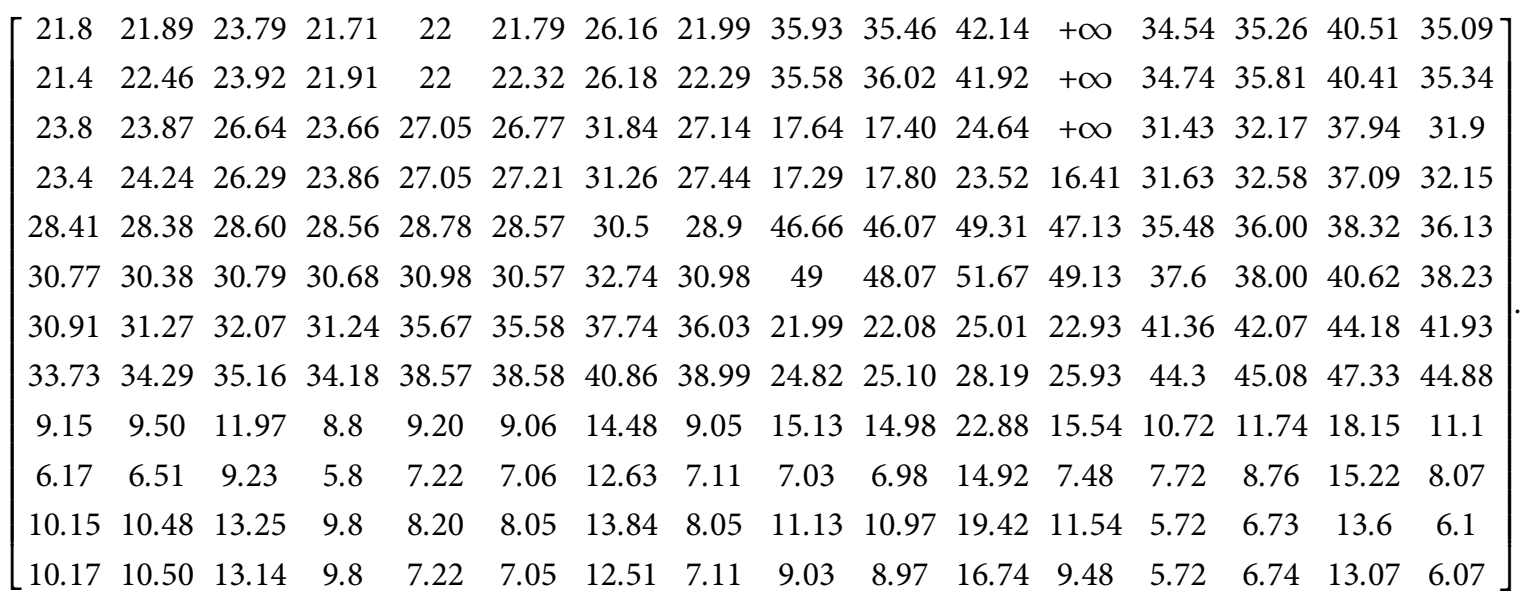


TABLE 2: Information on materials.

\begin{tabular}{|c|c|c|c|c|c|c|c|}
\hline \multirow{2}{*}{ Materials } & \multirow{2}{*}{ Weight $(t)$} & \multirow{2}{*}{ Time window $(\mathrm{h})\left[T W_{i m}^{\min }, T W_{i m}^{\max }\right]$} & \multirow{2}{*}{$\alpha_{i m}$} & \multicolumn{4}{|c|}{$\omega_{p}^{i m}$} \\
\hline & & & & $\omega_{1}^{i m}$ & $\omega_{2}^{i m}$ & $\omega_{3}^{i m}$ & $\omega_{4}^{i m}$ \\
\hline$r_{11}$ & 36 & {$[6,24]$} & 0.137 & 0.5 & 0.3 & 0.05 & 0.15 \\
\hline$r_{12}$ & 188 & {$[24,48]$} & 0.098 & 0.3 & 0.3 & 0.2 & 0.2 \\
\hline$r_{13}$ & 940 & {$[48,72]$} & 0.059 & 0.3 & 0.2 & 0.2 & 0.3 \\
\hline$r_{14}$ & 16 & {$[12,48]$} & 0.019 & 0.3 & 0.2 & 0.2 & 0.3 \\
\hline$r_{15}$ & 20 & {$[6,24]$} & 0.027 & 0.5 & 0.3 & 0.05 & 0.15 \\
\hline$r_{16}$ & 80 & {$[24,48]$} & 0.019 & 0.3 & 0.3 & 0.2 & 0.2 \\
\hline$r_{17}$ & 1200 & {$[48,72]$} & 0.004 & 0.3 & 0.2 & 0.2 & 0.3 \\
\hline$r_{24}$ & 8 & {$[12,52]$} & 0.012 & 0.4 & 0.2 & 0.1 & 0.3 \\
\hline$r_{31}^{24}$ & 34 & {$[6,24]$} & 0.191 & 0.5 & 0.3 & 0.05 & 0.15 \\
\hline$r_{32}$ & 150 & {$[24,48]$} & 0.082 & 0.3 & 0.3 & 0.2 & 0.2 \\
\hline$r_{33}$ & 1600 & {$[48,72]$} & 0.027 & 0.3 & 0.2 & 0.2 & 0.3 \\
\hline$r_{34}$ & $0\left(r_{34}=V_{4}\right)$ & {$[12,36]$} & 0.137 & 0.5 & 0.4 & 0.05 & 0.05 \\
\hline$r_{41}$ & 22 & {$[6,24]$} & 0.082 & 0.5 & 0.3 & 0.05 & 0.15 \\
\hline$r_{42}$ & 130 & {$[24,48]$} & 0.035 & 0.3 & 0.3 & 0.2 & 0.2 \\
\hline$r_{43}^{42}$ & 1500 & {$[48,72]$} & 0.012 & 0.3 & 0.2 & 0.2 & 0.3 \\
\hline$r_{44}$ & 10 & {$[12,36]$} & 0.059 & 0.4 & 0.3 & 0.2 & 0.1 \\
\hline
\end{tabular}

TABLE 3: Information on vehicles.

\begin{tabular}{|c|c|c|c|c|c|c|c|c|}
\hline \multirow{2}{*}{ Vehicles } & \multirow{2}{*}{ Cargo capacity (t) } & \multirow{2}{*}{ Loadable materials set } & \multirow[b]{2}{*}{$\xi_{j}$} & \multirow{2}{*}{ Vehicle type } & \multicolumn{4}{|c|}{$\omega_{q}^{j}$} \\
\hline & & & & & $\omega_{1}^{j}$ & $\omega_{2}^{j}$ & $\omega_{3}^{j}$ & $\omega_{4}^{j}$ \\
\hline$V_{1}$ & 20 & $R-\left\{r_{34}\right\},\left\{r_{34}\right\}$ & 0.6 & Truck & 0.3 & 0.2 & 0.25 & 0.25 \\
\hline$V_{2}$ & 20 & $R-\left\{r_{34}\right\}$ & 0.6 & Truck & 0.3 & 0.2 & 0.25 & 0.25 \\
\hline$V_{3}$ & 20 & $R-\left\{r_{34}\right\}$ & 0.5 & Truck & 0.3 & 0.2 & 0.25 & 0.25 \\
\hline$V_{4}$ & 2 & $\left\{r_{11}, r_{21}, r_{31}, r_{34}, r_{41}\right\}$ & 0.5 & Ambulance & 0.7 & 0.1 & 0.1 & 0.1 \\
\hline$V_{5}$ & 2000 & $R$ & 0.2 & Freight train & 0.2 & 0.4 & 0.2 & 0.2 \\
\hline$V_{6}$ & 2000 & $R$ & 0.2 & Freight train & 0.2 & 0.4 & 0.2 & 0.2 \\
\hline$V_{7}$ & 2000 & $R$ & 0.22 & Freight train & 0.2 & 0.4 & 0.2 & 0.2 \\
\hline$V_{8}$ & 2000 & $R$ & 0.22 & Freight train & 0.2 & 0.4 & 0.2 & 0.2 \\
\hline$V_{9}$ & 50 & $R$ & 0.1 & Helicopter & 0.3 & 0.4 & 0.15 & 0.15 \\
\hline$V_{10}$ & 50 & $R$ & 0.1 & Helicopter & 0.3 & 0.4 & 0.15 & 0.15 \\
\hline$V_{11}$ & 50 & $R$ & 0.1 & Helicopter & 0.3 & 0.4 & 0.15 & 0.15 \\
\hline$V_{12}$ & 50 & $R$ & 0.1 & Helicopter & 0.3 & 0.4 & 0.15 & 0.15 \\
\hline
\end{tabular}

The maximum positive deviation of $T_{i m}^{j}$ is as follows:

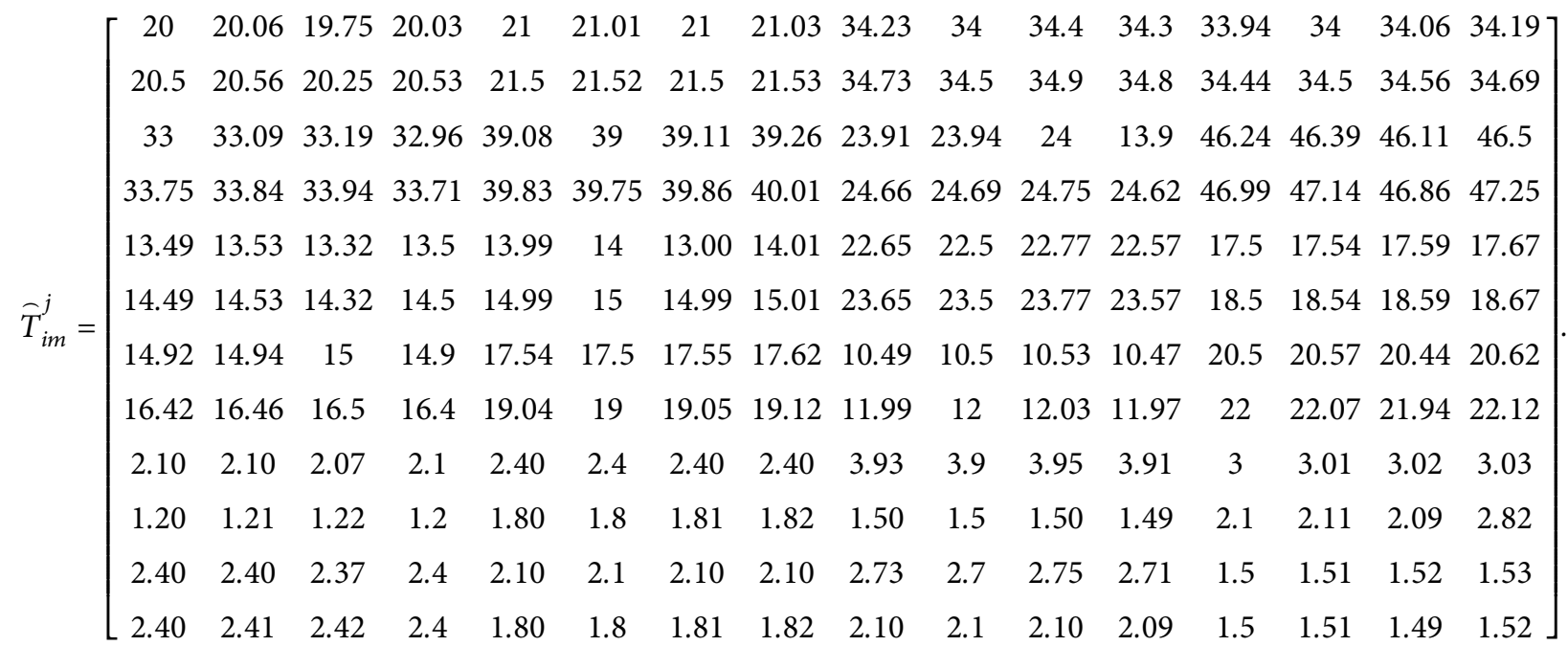


The distances $d_{i m}^{j}$ between $r_{i m}$ and $V_{j}$ are as follows:

$$
d_{i m}^{j}=\left[\begin{array}{cccccccccccccccc}
40 & 45 & 20 & 42 & 21 & 22 & 21 & 23 & 130 & 112 & 144 & 120 & 70 & 75 & 80 & 90 \\
40 & 45 & 20 & 42 & 21 & 22 & 21 & 23 & 130 & 112 & 144 & 120 & 70 & 75 & 80 & 90 \\
30 & 35 & 40 & 28 & 40 & 36 & 42 & 50 & 20 & 22 & 25 & 18 & 65 & 73 & 58 & 79 \\
30 & 35 & 40 & 28 & 40 & 36 & 42 & 50 & 20 & 22 & 25 & 18 & 65 & 73 & 58 & 79 \\
58 & 63 & 38 & 60 & 39 & 40 & 39 & 41 & 148 & 130 & 162 & 138 & 88 & 93 & 98 & 108 \\
58 & 63 & 38 & 60 & 39 & 40 & 39 & 41 & 148 & 130 & 162 & 138 & 88 & 93 & 98 & 108 \\
50 & 55 & 60 & 48 & 60 & 56 & 62 & 70 & 40 & 42 & 45 & 38 & 85 & 93 & 78 & 99 \\
50 & 55 & 60 & 48 & 60 & 56 & 62 & 70 & 40 & 42 & 45 & 38 & 85 & 93 & 78 & 99 \\
80 & 85 & 60 & 82 & 61 & 62 & 61 & 63 & 170 & 152 & 184 & 160 & 110 & 115 & 120 & 130 \\
60 & 65 & 70 & 58 & 70 & 66 & 72 & 80 & 50 & 52 & 55 & 48 & 95 & 103 & 88 & 109 \\
85 & 90 & 65 & 87 & 66 & 67 & 66 & 68 & 175 & 157 & 189 & 165 & 115 & 120 & 125 & 135 \\
80 & 80 & 90 & 78 & 90 & 86 & 92 & 100 & 70 & 72 & 75 & 68 & 115 & 123 & 108 & 129
\end{array}\right] .
$$

The handling $t_{i m}^{j}$ time is as follows:

$$
t_{i m}^{j}=\left[\begin{array}{cccccccccccccccc}
1.8 & 1.82 & 4.04 & 2.24 & 1 & 0.78 & 5.16 & 0.96 & 1.7 & 1.46 & 7.74 & +\infty & 1.1 & 1.26 & 6.45 & 0.9 \\
0.9 & 1.90 & 3.67 & 1.84 & 0.5 & 0.81 & 4.68 & 0.76 & 0.85 & 1.52 & 7.02 & +\infty & 0.55 & 1.31 & 5.85 & 0.65 \\
1.8 & 1.80 & 4.51 & 2.24 & 1 & 0.77 & 5.76 & 0.96 & 1.7 & 1.44 & 8.64 & +\infty & 1.1 & 1.25 & 7.2 & 0.9 \\
0.9 & +\infty & +\infty & +\infty & 0.5 & +\infty & +\infty & +\infty & 0.85 & 0.85 & 0.85 & 0 & 0.55 & +\infty & +\infty & +\infty \\
1.44 & 1.33 & 1.97 & 2.08 & 0.8 & 0.57 & 2.52 & 0.88 & 1.36 & 1.07 & 3.78 & 2 & 0.88 & 0.92 & 3.15 & 0.8 \\
1.8 & 1.33 & 2.16 & 2.24 & 1 & 0.57 & 2.76 & 0.96 & 1.7 & 1.07 & 4.14 & 2 & 1.1 & 0.92 & 3.45 & 0.9 \\
1.08 & 1.35 & 2.07 & 1.92 & 0.6 & 0.58 & 2.64 & 0.8 & 1.02 & 1.08 & 3.96 & 2 & 0.66 & 0.94 & 3.3 & 0.7 \\
0.9 & 1.37 & 2.16 & 1.84 & 0.5 & 0.58 & 2.76 & 0.76 & 0.85 & 1.10 & 4.14 & 2 & 0.55 & 0.95 & 3.45 & 0.65 \\
2.16 & 2.48 & 5.08 & 2.4 & 1.2 & 1.06 & 6.48 & 1.04 & 2.04 & 1.98 & 9.72 & 2.5 & 1.32 & 1.72 & 8.1 & 1 \\
2.16 & 2.48 & 5.17 & 2.4 & 1.2 & 1.06 & 6.6 & 1.04 & 2.04 & 1.98 & 9.9 & 2.5 & 1.32 & 1.72 & 8.25 & 1 \\
2.16 & 2.46 & 5.36 & 2.4 & 1.2 & 1.05 & 6.84 & 1.04 & 2.04 & 1.97 & 10.26 & 2.5 & 1.32 & 1.70 & 8.55 & 1 \\
2.16 & 2.46 & 5.08 & 2.4 & 1.2 & 1.05 & 6.48 & 1.04 & 2.04 & 1.97 & 9.72 & 2.5 & 1.32 & 1.70 & 8.1 & 1
\end{array}\right] .
$$

And, the loading conditions' satisfaction $u_{3}^{i m, j}$ is as follows:

$$
u_{3}^{i m, j}=\left[\begin{array}{cccccccccccccccc}
0.5 & 0.75 & 1 & 0.5 & 0.5 & 0.75 & 1 & 0.5 & 0.5 & 0.75 & 1 & 0 & 0.5 & 0.75 & 1 & 0.5 \\
1 & 1 & 0.5 & 1 & 1 & 1 & 0.5 & 1 & 1 & 1 & 0.5 & 0 & 1 & 1 & 0.5 & 1 \\
0.5 & 0.75 & 1 & 0.5 & 0.5 & 0.75 & 1 & 0.5 & 0.75 & 0.75 & 1 & 0 & 0.5 & 0.75 & 1 & 0.5 \\
1 & 0 & 0 & 0 & 1 & 0 & 0 & 0 & 1 & 0 & 0 & 1 & 1 & 0 & 0 & 0 \\
0.75 & 1 & 1 & 0.75 & 0.75 & 1 & 1 & 0.75 & 0.75 & 1 & 1 & 0 & 0.75 & 1 & 1 & 0.75 \\
0.75 & 1 & 1 & 0.75 & 0.75 & 1 & 1 & 0.75 & 0.75 & 1 & 1 & 0 & 0.75 & 1 & 1 & 0.75 \\
0.75 & 1 & 1 & 0.75 & 0.75 & 1 & 1 & 0.75 & 0.75 & 1 & 1 & 0 & 0.75 & 1 & 1 & 0.75 \\
0.75 & 1 & 1 & 0.75 & 0.75 & 1 & 1 & 0.75 & 0.75 & 1 & 1 & 0 & 0.75 & 1 & 1 & 0.75 \\
1 & 0.75 & 0.5 & 1 & 1 & 0.75 & 0.75 & 1 & 1 & 0.75 & 0.5 & 0 & 1 & 0.75 & 0.5 & 1 \\
1 & 0.75 & 0.5 & 1 & 1 & 0.75 & 0.75 & 1 & 1 & 0.75 & 0.5 & 0 & 1 & 0.75 & 0.5 & 1 \\
1 & 0.75 & 0.5 & 1 & 1 & 0.75 & 0.75 & 1 & 1 & 0.75 & 0.5 & 0 & 1 & 0.75 & 0.5 & 1 \\
1 & 0.75 & 0.5 & 1 & 1 & 0.75 & 0.75 & 1 & 1 & 0.75 & 0.5 & 0 & 1 & 0.75 & 0.5 & 1
\end{array}\right] .
$$


5.1. Example Results. Setting $\varepsilon=10^{-8}$ and $\kappa=10 \%$, we calculate the satisfaction value matrices of $R_{V}^{\prime}=\left(\lambda_{j i m}^{\prime}\right)_{J \times I \times M}$ and $V_{R}=\left(\theta_{j i m}\right)_{J \times I \times M}$ are as follows:

\begin{tabular}{|c|c|c|c|c|c|c|c|c|c|c|c|c|c|c|c|c|}
\hline & 0 & 0 & 0 & 0.0078 & 0.0087 & 0 & 0 & 0.005 & 0 & 0 & 0 & 0 & 0 & 0 & 0 & 0.0149 \\
\hline & 0 & 0 & 0 & 0.0089 & 0.009 & 0 & 0 & 0.0053 & 0 & 0 & 0 & 0 & 0 & 0 & 0 & 0.017 \\
\hline & 0 & 0 & 0 & 0.0079 & 0.0083 & 0 & 0 & 0.0047 & 0 & 0 & 0 & 0 & 0 & 0 & 0 & 0.0163 \\
\hline & 0 & 0 & 0 & 0 & 0 & 0 & 0 & 0 & 0 & 0 & 0 & 0.0571 & 0 & 0 & 0 & 0 \\
\hline & 0.0511 & 0.0512 & 0.0366 & 0.0097 & 0.0108 & 0.0101 & 0.0027 & 0.0058 & 0.0594 & 0.0378 & 0.0164 & 0 & 0.0264 & 0.0172 & 0.0076 & 0.0222 \\
\hline & 0.0491 & 0.0502 & 0.0358 & 0.0095 & 0.0104 & 0.0099 & 0.0026 & 0.0056 & 0.0579 & 0.0373 & 0.0162 & 0 & 0.0256 & 0.0169 & 0.0074 & 0.0217 \\
\hline & 0.0476 & 0.0483 & 0.0347 & 0.0092 & 0.0095 & 0.0092 & 0.0025 & 0.0052 & 0.084 & 0.0458 & 0.0197 & 0 & 0.0237 & 0.0159 & 0.0071 & 0.0203 \\
\hline & 0.0456 & 0.047 & 0.0337 & 0.009 & 0.0091 & 0.009 & 0.0024 & 0.005 & 0.0791 & 0.0441 & 0.0191 & 0 & 0.0229 & 0.0156 & 0.007 & 0.0198 \\
\hline & 0.1125 & 0 & 0 & 0.0168 & 0.0239 & 0 & 0 & 0.0109 & 0.1302 & 0 & 0 & 0 & 0.0599 & 0 & 0 & 0.0463 \\
\hline & 0.137 & 0 & 0 & 0.019 & 0.027 & 0 & 0 & 0.012 & 0.191 & 0 & 0 & 0 & 0.0706 & 0 & 0 & 0.0526 \\
\hline & 0.1068 & 0 & 0 & 0.0162 & 0.0253 & 0 & 0 & 0.0114 & 0.1518 & 0 & 0 & 0 & 0.082 & 0 & 0 & 0.0589 \\
\hline & 0.1067 & 0 & 0 & 0.0162 & 0.027 & 0 & 0 & 0.012 & 0.1685 & 0 & 0 & 0 & 0.082 & 0 & 0 & 0.059 \\
\hline & 0 & 0 & 0 & 0.8955 & 0 & 0 & 0 & 0.9367 & 0 & 0 & 0 & 0 & 0 & 0 & 0 & 0 \\
\hline & 0 & 0 & 0 & 0.8924 & 0 & 0 & 0 & 0.933 & 0 & 0 & 0 & 0 & 0 & 0 & 0 & 0 \\
\hline & 0 & 0 & 0 & 0.8498 & 0 & 0 & 0 & 0.7187 & 0 & 0 & 0 & 0 & 0 & 0 & 0 & 0 \\
\hline & 0 & 0 & 0 & 0 & 0 & 0 & 0 & 0 & 0 & 0 & 0 & 0.8821 & 0 & 0 & 0 & 0 \\
\hline & 0 & 0.8446 & 0.8912 & 0.7103 & 0 & 0.9676 & 0.8266 & 0.8705 & 0 & 0 & 0.001 & 0 & 0 & 0.6314 & 0.5541 & 0 \\
\hline & 0 & 0.83 & 0.8885 & 0.6748 & 0 & 0.9439 & 0.8241 & 0.8341 & 0 & 0 & 0.001 & 0 & 0 & 0.5775 & 0.5525 & 0 \\
\hline & 0 & 0.7827 & 0.7444 & 0.6857 & 0 & 0.7223 & 0.6782 & 0.5563 & 0.3414 & 0.9424 & 0.0952 & 0.7811 & 0 & 0.2142 & 0.4707 & 0 \\
\hline & 0 & 0.7247 & 0.7402 & 0.6324 & 0 & 0.6248 & 0.6744 & 0.5034 & 0 & 0.9357 & 0.0952 & 0.6938 & 0 & 0.1085 & 0.4673 & 0 \\
\hline & 0.8928 & 0 & 0 & 0.9009 & 0.971 & 0 & 0 & 0.9896 & 0.3213 & 0 & 0 & 0.4957 & 0.7689 & 0 & 0 & 0.7171 \\
\hline & 0.897 & 0 & 0 & 0.9073 & 0.8303 & 0 & 0 & 0.7422 & 0.966 & 0 & 0 & 0.9727 & 0.5482 & 0 & 0 & 0.01 \\
\hline & 0.8795 & 0 & 0 & 0.9024 & 0.9827 & 0 & 0 & 0.9896 & 0.3868 & 0 & 0 & 0.5049 & 0.8092 & 0 & 0 & 0.7171 \\
\hline & 0.8629 & 0 & 0 & 0.9068 & 0.8302 & 0 & 0 & 0.7422 & 0.9489 & 0 & 0 & 0.9721 & 0.5519 & 0 & 0 & 0.01 \\
\hline
\end{tabular}

Assuming $\lambda^{\prime}=10^{-7}$ and $\theta^{\prime}=10^{-7}$ and using improved NIMP algorithm to solve the model, finally, we get the maximum weighted satisfaction of $F 1$ is 0.7392 , and the optimal matching result is shown in Table 4.

It can be seen from the result that the loading characteristics are considered in the allocation scheme so that the materials can be matched with particular vehicles. This scheme is more specific than the existing research and plays a more practical guiding role in material emergency transportation.

5.2. Example Analysis. Considering the uncertainty of arrival time in emergency transportation, we design the following experiments to show that the matching scheme still has certain reliability under different delay risks.

(1) Firstly, set $\kappa=10 \%$, and the delay probability $\xi_{j}$ of $V_{j}$ is given in Table 3 . Assume in actual rescue, the disturbance coefficient $\kappa_{\text {actual }}$ is $20 \%$, and the delay time of the delayed vehicle is $\kappa_{\text {actual }}$ $* T_{i m}^{j}=20 \% * T_{i m}^{j}$. In this condition, we calculate the satisfaction of the optimal scheme attacked by disturbance coefficient $\kappa_{\text {actual }}\left(\kappa_{\text {actual }}\right.$-optimal scheme for short) and the scheme in Table 4 ( $\kappa$-current scheme for short), respectively. Repeat this experiment 2000 times, and the comparison between the $\kappa$-current and the $\kappa_{\text {actual }}$-optimal scheme is shown in Figure 2. We use green circle which denotes $\kappa$-current scheme satisfaction and blue line which denotes $\kappa_{\text {actual }}{ }^{-o p-}$ timal scheme satisfaction. It can be seen from Figure 2 , when $\kappa=10 \%$ and disturbance coefficient $\kappa_{\text {actual }} l$ is $20 \%$, the gap between the $\kappa$-current scheme and the $\kappa_{\text {actual }}$-optimal scheme is not noticeable. This result shows that $\kappa=10 \%$ and can make the scheme stable to a certain extent.

(2) On the basis of the above experiment, we still make $\kappa=10 \%$ and change the value of disturbance coefficient $\kappa_{\text {actual }}$ from 0 to $70 \%$. Under each value of 
TABLE 4: Information on vehicles.

\begin{tabular}{|c|c|c|}
\hline Area & Vehicle type & Result \\
\hline$R 1$ & $\begin{array}{c}\text { Helicopter } \\
\text { Freight train }\end{array}$ & $\begin{array}{c}\left\{V_{9}\right\} \longleftrightarrow\left\{r_{11}\right\} \\
\left\{V_{5}\right\} \stackrel{\leftrightarrow}{\longleftrightarrow}\left\{r_{12}, r_{13}, r_{14}\right\}\end{array}$ \\
\hline$\underline{R} 2$ & $\begin{array}{c}\text { Helicopter } \\
\text { Freight train }\end{array}$ & $\begin{array}{l}\left\{V_{12}\right\} \longleftrightarrow\left\{r_{21}, r_{24}\right\} \\
\left\{V_{8}\right\} \longleftrightarrow\left\{r_{22}, r_{23}\right\}\end{array}$ \\
\hline R3 & $\begin{array}{l}\text { Helicopter } \\
\text { Freight train } \\
\text { Ambulance }\end{array}$ & $\begin{array}{c}\left\{V_{10}\right\} \longleftrightarrow\left\{r_{31}\right\} \\
\left\{V_{7}\right\} \longleftrightarrow\left\{r_{32}, r_{33}\right\} \\
\left\{V_{4}\right\} \longleftrightarrow\left\{r_{34}\right\}\end{array}$ \\
\hline$R 4$ & $\begin{array}{c}\text { Helicopter } \\
\text { Freight train }\end{array}$ & $\begin{array}{l}\left\{V_{11}\right\} \longleftrightarrow\left\{r_{41}, r_{44}\right\} \\
\left\{V_{6}\right\} \longleftrightarrow\left\{r_{42}, r_{43}\right\}\end{array}$ \\
\hline
\end{tabular}

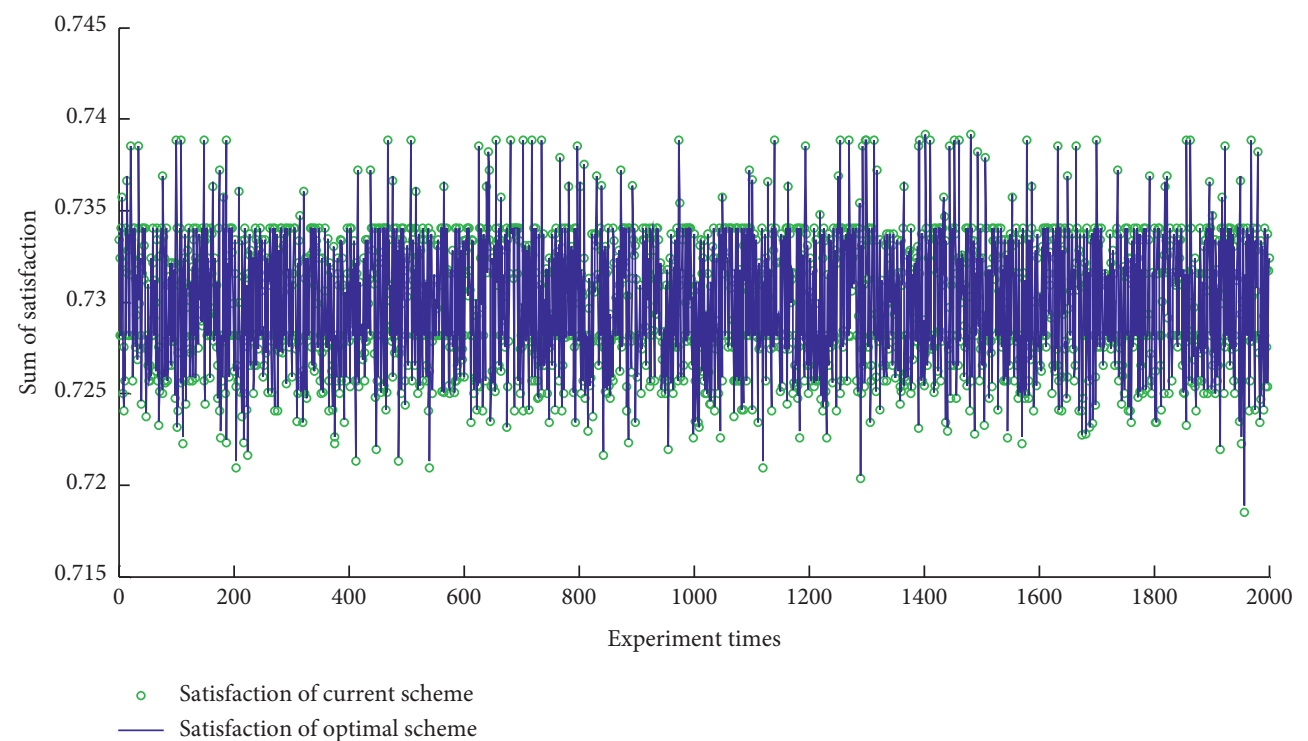

FIGURE 2: Comparison between the $\kappa$-current and the $\kappa_{\text {actual }}$-optimal schemes.

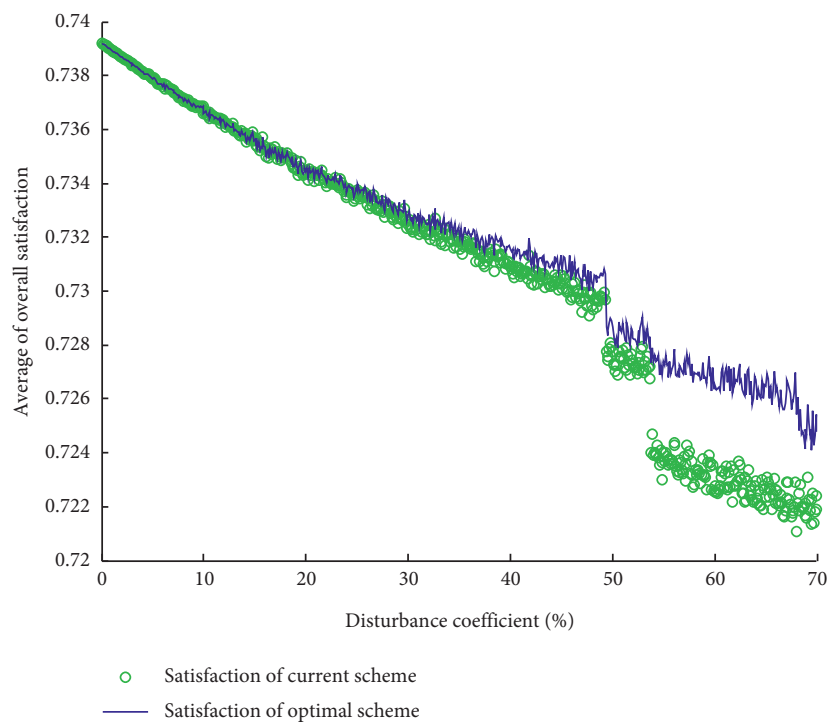

Figure 3: Relationship between the gap and the disturbance coefficient. 


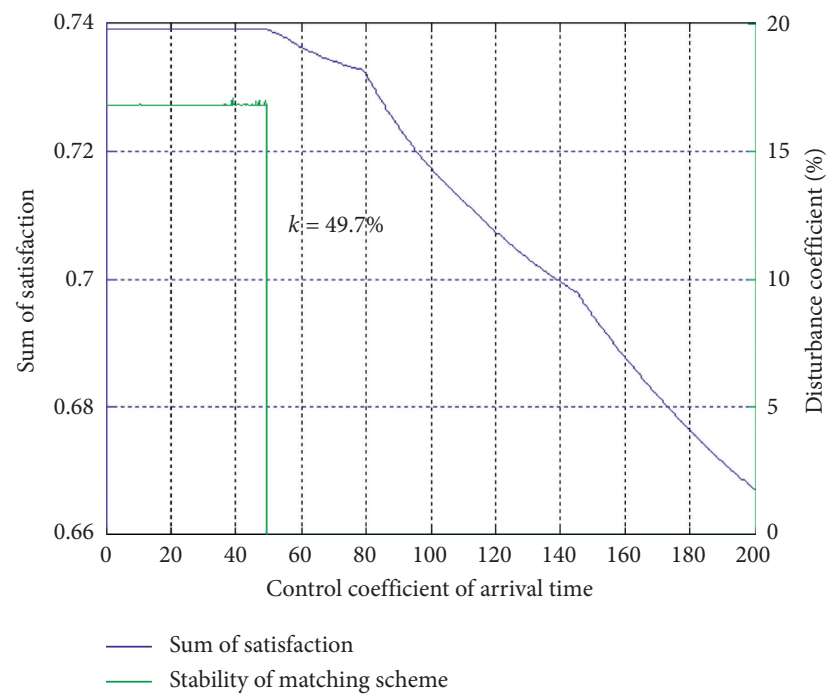

FIgURE 4: Relationship between $\kappa$ and the satisfaction of schemes, and the relationship between $\kappa$ and stability of the $\kappa$-current schemes.

$\kappa_{\text {actual }}$, we calculate the mean satisfaction value of the $\kappa$-current and $\kappa_{\text {actual }}$-optimal schemes. The comparison is shown in Figure 3.

We use the green circle which denotes the variation in $\kappa$-current scheme satisfaction and the blue line which denotes the variation in $\kappa_{\text {actual }}$-optimal scheme satisfaction. The result shows that, with the increase of the disturbance coefficient $\kappa_{\text {actual }}$, the average satisfaction of the $\kappa$-current schemes and the $\kappa_{\text {actual }}$-optimal schemes show a downward trend, and the descent speed of $\kappa$-current schemes is faster than $\kappa_{\text {actual }}$-optimal schemes. Even so, when the disturbance coefficient is less than $53.7 \%$, the difference between the satisfaction of the $\kappa$-current schemes and the $\kappa_{\text {actual }}$-optimal schemes is still not significant. When the disturbance coefficient exceeds $53.7 \%$, because the overall satisfaction of $R 3$ decrease significantly, the gap between the $\kappa$-current schemes and the $\kappa_{\text {actual }}$-optimal schemes has been widened.

(3) The value of $\kappa$ not only affects the reliability of the scheme but also affects the satisfaction of the $\kappa$-current scheme, so we change $\kappa$ from 0 to $200 \%$ to find a reasonable value. Figure 4 shows the relationship between $\kappa$ and the satisfaction of the $\kappa$-current scheme (green line) and the relationship between $\kappa$ and stability of matching scheme (blue line) as well.

The green line expresses if $\kappa \leq 49.7 \%$, and the satisfaction of the $\kappa$-current schemes remains unchanged. Once $\kappa>49.7 \%$, the satisfaction of $\kappa$-current schemes falls rapidly.

The blue line shows the value of disturbance coefficient $\kappa_{\text {actual }}$ that the satisfaction of the $\kappa$-current scheme is lower than that of the optimal scheme for the first time. When $\kappa \leq 49.7 \%$, the value remains almost unchanged.
When $\kappa>49.7 \%$, because of the nonideal $\kappa$-current scheme, all the optimal schemes are better than the $\kappa$-current ones.

Based on the above analysis, the value of $\kappa$ should not exceed $49.7 \%$.

In practical application, the value of $\kappa$ can also be analyzed according to the specific situation of vehicles and materials to let the scheme have better stability and operability.

\section{Summary}

This paper studies the matching problem between the materials to be transported and the comprehensive transportation network emergency vehicles in the process of rescue, aiming to promote the information sharing of transportation resources and the rational allocation of transportation resources and improve the quality of emergency rescue. We first establish evaluation index systems to measure the satisfaction of both parties. Next, based on the realistic constraints of the materials and vehicles matching, the multiobjective optimization model is established to maximize the satisfaction of the matching parties. Then, an improved NIMP algorithm is designed to solve the model. Finally, through the calculation of an example, it shows that the proposed method can obtain the optimal matching scheme different from the existing literature, which can specify the materials transported by certain vehicles on the basis of loading and arrival time demand. At the same time, repeated experiments show that the obtained matching scheme has excellent stability in the face of the uncertainty of transportation time caused by emergencies.

The limitations of the paper are as follows. (1) The situation of multimodal transportation is not considered. (2) It is not applicable that materials can be separated. (3) Discussion about blending loading demand for materials is 
lacking. Therefore, in the next step of research, it is attemptable to transform the one-to-many matching mode into many-to-many matching mode and improve the satisfaction index extraction based on the characteristics of emergency.

\section{Data Availability}

The data used to support the findings of this study are available from the corresponding author upon request.

\section{Conflicts of Interest}

The authors declare that there are no conflicts of interest regarding the publication of this paper.

\section{Acknowledgments}

This research was supported by the National Key R\&D Program of China (2017YFB1200702), National Natural Science Foundation of China (Project no. 52072314), Sichuan Science and Technology Program (Project nos. 2020YFH0035, 2020YJ0268, 2020YJ0256, and 2020JDRC0032), Chengdu Science and Technology Plan Research Program (Project nos. 2019YF0501493SN and 2020RK0000036ZF), Natural Science Foundation of Zhejiang Province, China (LQ18G030012), and Humanities and Social Sciences Fund of Ministry of Education, China (18YJC630190).

\section{References}

[1] L. Y. Zhang, T. Fei, J. Zhang, and J. Li, "Application of emergency logistics distribution routing optimization based on improved ant colony algorithm," Advanced Materials Research, vol. 268-270, pp. 1726-1732, 2011.

[2] Z.-H. Hu, "A container multimodal transportation scheduling approach based on immune affinity model for emergency relief," Expert Systems with Applications, vol. 38, no. 3, pp. 2632-2639, 2011.

[3] L. Özdamar and O. Demir, "A hierarchical clustering and routing procedure for large scale disaster relief logistics planning," Transportation Research Part E: Logistics and Transportation Review, vol. 48, no. 3, pp. 591-602, 2012.

[4] C. G. Rawls and M. A. Turnquist, "Pre-positioning and dynamic delivery planning for short-term response following a natural disaster," Socio-Economic Planning Sciences, vol. 46, no. 1, pp. 46-54, 2012.

[5] A. Bozorgi-Amiri, M. S. Jabalameli, and S. M. J. Mirzapour Ale-Hashem, A Multi-Objective Robust Stochastic Programming Model for Disaster Relief Logistics Under Uncertainty, SpringVerlag, New York, NY, USA, 2013.

[6] A. Afshar and A. Haghani, "Modeling integrated supply chain logistics in real-time large-scale disaster relief operations," Socio-Economic Planning Sciences, vol. 46, no. 4, pp. 327-338, 2012.

[7] F. Sabouhi, M. Heydari, and A. Bozorgi-Amiri, "Multi-objective routing and scheduling for relief distribution with spilt delivery in post-disaster response," Journal of Industrial and Systems Engineering, vol. 9, no. 3, pp. 17-27, 2016.

[8] L. Huang, J. Yang, and C.-H. Chen, "An improved swarm intelligence algorithm for multi-Item joint ordering strategy of cruise ship supply," Mathematical Problems in Engineering, vol. 2020, Article ID 5048629, 9 pages, 2020.

[9] G. Laporte, F. Louveaux, and H. Mercure, "Models and exact solutions for a class of stochastic location-routing problems," European Journal of Operational Research, vol. 39, no. 1, pp. 71-78, 1989.

[10] G. Barbarosoglu and Y. Arda, "A two-stage stochastic programming framework for transportation planning in disaster response phase," Journal of the Operational Research Society, vol. 55, no. 1, pp. 43-53, 2004.

[11] X. Duan, S. Song, and J. Zhao, "Emergency vehicle dispatching and redistribution in highway network based on bilevel programming," Mathematical Problems in Engineering, vol. 2015, Article ID 731492, 12 pages, 2015.

[12] W. Klibi, S. Ichoua, and A. Martel, "Prepositioning emergency supplies to support disaster relief: a case study using stochastic programming," Information Systems and Operational Research, vol. 1, no. 56, pp. 50-80, 2018.

[13] D. Gale and L. S. Shapley, "College admissions and the stability of marriage," The American Mathematical Monthly, vol. 69, no. 1, pp. 9-15, 1962.

[14] A. E. Roth, "Common and conflicting interests in two-sided matching markets," European Economic Review, vol. 27, no. 1, pp. 75-96, 1985.

[15] A. E. Roth, "On the allocation of residents to rural hospitals: a general property of two-sided matching markets," Econometric a, vol. 54, no. 54, pp. 425-427, 1986.

[16] D. He, C. Cui, and J. Guo, "Research on the selection of military emergency material reserve," Mathematics in Practice and Theory, vol. 49, no. 15, pp. 61-68, 2019.

[17] J. Li, X. Yao, D. Chen, and S. Ni, "Evaluation index selection of comprehensive inter-regional traffic emergency rescue plan," China Safety Science Journal, vol. 28, no. S2, pp. 185-190, 2018.

[18] D. Chen, Y. Sun, J. Li, and S. Ni, "Construction of evaluation index system for emergency rescue capacity of rail transit under serious epidemic situation," Journal of Traffic and Transportation Engineering, vol. 20, no. 3, pp. 129-138, 2020.

[19] L. Su, C. Yin, D. Chen, H. Lv, and Q. Zhang, "Cascading failure in multiple critical infrastructure interdependent networks of syncretic railway system," IEEE Transactions on Intelligent Transportation Systems, pp. 1-14, 2021.

[20] T. Yang, X. Peng, D. Chen, F. Yang, and M. Muneeb Abid, "Research on trans-region integrated traffic emergency dispatching technology based on multi-agent," Journal of Intelligent \& Fuzzy Systems, vol. 38, no. 5, pp. 5763-5774, 2020.

[21] J. Zhu, R. Wang, and Y. Li, "A decision method of vehicle cargo bilateral matching based on the information of uncertainty language relevance," Journal of Systems Science, vol. 26, no. 1, pp. 86-91, 2018.

[22] Z. Wang, Y. Li, F. Gu, J. Guo, and X. Wu, "Two-sided matching and strategic selection on freight resource sharing platforms," Physica, A. Statistical Mechanics and its Applications, vol. 559, pp. 1-18, 2020.

[23] B. Yang, X. Ye, R. Wang, and B. Shuai, "Method for vehiclecargo two-sided fair matching based on intuitionistic Fuzzy optimization," Computer Integrated Manufacturing Systems, pp. 1-14, 2021.

[24] B. Wang, J. Cui, and D. Kong, "Research on the decisionmaking method of vehicle-cargo two-sided matching based on grey absolute incidence degree," Value Engineering, vol. 38, no. 23, pp. 122-125, 2019.

[25] Y. Gu, M. Su, L. Zhang, and Y. Zheng, "Research on vehicle and goods attributes matching of platform based on bidding 
and order distribution mode," Journal of Wuhan University of Technology (Transportation Science \&. Engineering), vol. 44, no. 3, pp. 450-455, 2020.

[26] Z.-S. Chen, X.-L. Liu, K.-S. Chin, W. Pedrycz, K.-L. Tsui, and M. J. Skibniewski, "Online-review analysis based large-scale group decision-making for determining passenger demands and evaluating passenger satisfaction: case study of highspeed rail system in China," Information Fusion, vol. 69, pp. 22-39, 2021.

[27] Z.-S. Chen, X.-L. Liu, R. M. Rodriguez et al., "Identifying and prioritizing factors affecting in-cabin passenger comfort on high-speed rail in China: a fuzzy-based linguistic approach," Applied Soft Computing, vol. 59, pp. 1-18, 2020.

[28] A. E. Roth, "The evolution of the labor market for medical interns and residents: a case study in game theory," Journal of Political Economy, vol. 92, no. 6, pp. 991-1016, 1984. 